\title{
Sleep-related breathing disorders: impact on mortality of cerebrovascular disease
}

\author{
O. Parra*, A. Arboix\# , J.M. Montserrat ${ }^{\Uparrow}$, L. Quintó ${ }^{+}$, S. Bechich*, L. García-Eroles*
}

Sleep-related breathing disorders: impact on mortality of cerebrovascular disease. O. Parra, A. Arboix, J.M. Montserrat, L. Quintó, S. Bechich, L. García-Eroles. (C) ERS Journals Ltd 2004.

ABSTRACT: The aim of the study was to analyse the impact of sleep-related breathing disorders in a 2-yr survival follow-up of patients with a first ever stroke or transient ischaemic attack.

The study followed 161 patients. Complete neurological assessment was performed in order to determine cerebrovascular risk factors, functional disability, and parenchymatous and vascular localisation, as well as stroke subtype categorisation. A sleep study was carried out using a portable respiratory recording device. The entire cohort was followed over a mean period of $\mathbf{2 2 . 8}$ months. The main outcome event was death and time of survival since the neurological event. A multivariate Cox's model was estimated.

The patients were ages $72 \pm 9$ yrs (mean \pm SD), and had a body mass index of $26.6 \pm 3.9 \mathrm{~kg} \cdot \mathrm{m}^{-2}$ and apnoea/hypopnoea index (AHI) of $21.2 \pm 15.7$. Overall, mortality occurred in 22 cases, and the survival rate was $86.3 \%$. Vascular disease accounted for $63.6 \%$ of deaths. Multivariate analysis selected four independent variables associated with mortality: 1) age; 2) AHI, with an implied 5\% increase in mortality risk for each additional unit of $\mathrm{AHI} ; 3$ ) involvement of the middle cerebral artery; and 4) the presence of coronary disease.

In conclusion, the findings suggest that sleep-related breathing disorders are an independent prognostic factor related to mortality after a first episode of stroke. Eur Respir J 2004; 24: 267-272.
Depts of *Pneumology and ${ }^{*}$ Neurology, Hospital del Sagrat Cor, Institut Clínic de Pneumologia i Cirurgia Toràcica, and ${ }^{+}$Unitat d'Epidemiologia i Bioestadística, Hospital Clínic, University of Barcelona, Barcelona, Spain.

Correspondence: O. Parra, Servei de Pneumologia, Hospital del Sagrat Cor, Viladomat 288, 08029 Barcelona, Spain.

Fax: 34934948906

E-mail: 22515opo@comb.es

Keywords: Cerebrovascular disorders, mortality, sleep apnoea/hypopnoea syndrome

Received: June 22003

Accepted after revision: March 12004

This study was supported by grants from the Sociedad Española de Patologia del Aparato Respiratorio (Barcelona, Spain) in 1995 and 2001 and by grant RR-ISCIII-RTCI (Instituto Carlos III, Madrid, Spain).
Increasing evidence suggests that snoring and sleep apnoea syndrome (SAS) are associated with cerebrovascular disease (CVD), either as a risk factor [1-4] or as a consequence of certain neurological locations [5-7]. A number of case/control studies have shown a significantly higher frequency of snoring and/or apnoeas in patients with CVD, suggesting that this condition acts as a risk factor [1-3, 8-11]. However, some doubts have been raised about the relevance of SAS to public health [12], mainly because some conditions, such as ageing and obesity, could act as confounding factors. Nevertheless, some studies have regarded SAS as a risk factor for cardiovascular morbidity [13, 14] and mortality [15-18]. The existence of a high prevalence of sleep-related breathing disorders (SRBDs) in patients with a first ever stroke or transient ischaemic attack (TIA) was recently confirmed [19]. This prevalence was greater than expected, considering the available epidemiological data in Spain. The data also suggest that obstructive events act as a risk factor, whereas central events and Cheyne-Stokes respiration (CSR) could be the consequence of CVD [19]. In this regard, NETZER et al. [20] recently demonstrated a significant decline in the blood flow of the middle cerebral artery occurring during obstructive apnoeas and hypopnoeas, but not during central events. These observations account for the pathophysiological basis whereby obstructive events could be a risk factor in acute CVD. However, some issues such as the identification of SRBDs as a long-term prognostic factor in CVD are essential

For editorial comments see page 195. for definitively establishing the impact of SRBDs on health Therefore, the aim of the present study was to analyse the influence of SRBDs on mortality over 2 yrs in patients with a first ever stroke or TIA.

\section{Materials and methods}

Patients with a first-ever stroke or TIA ( $\mathrm{n}=161 ; 82$ males), who were admitted to the stroke unit of the Sagrat Cor Hospital (Barcelona, Spain) and included in the stroke registry [21], were studied. Only one patient declined enrolment. The protocol was approved by the human ethics committee of the Sagrat Cor Hospital and informed consent was obtained from all of the patients or their families.

Complete neurological assessment was performed. The following cerebrovascular risk factors or comorbid conditions were recorded: demographic characteristics (age and sex), history of hypertension, diabetes, myocardial infarction or angina, rheumatic heart disease, congestive heart failure, atrial fibrillation, smoking $\left(>20\right.$ cigarettes $\cdot$ day $\left.^{-1}\right)$, alcohol abuse $\left(>80 \mathrm{~g}\right.$ alcohol $\left.\cdot \mathrm{day}^{-1}\right)$, and chronic obstructive pulmonary disease; vascular risk factors have been defined elsewhere [22]. The salient features of the clinical and neurological examination and results of routine laboratory tests (blood cell count, biochemical profile, serum electrolyte levels and urinalysis), chest radiography and 12-lead electrocardiography were recorded. Functional abilities were assessed using the Barthel index. The maximal severity of stroke or 
neurological impairment was estimated using the Canadian scale [23]. Stroke subtypes were also categorised according to the Cerebrovascular Study Group of the Spanish Society of Neurology, which is similar to the National Institute of Neurological Disorders and Stroke classification [24]. The following subtypes were considered: TIA; ischaemic stroke, either atherothrombotic, cardioembolic, lacunar, unusual or of undetermined origin; and intraparenchymatous haemorrhagic stroke. Stroke parenchymatous topography, such as hemispheric (identifying different areas), brain stem and cerebellar location, was classified on the basis of brain computed tomography or magnetic resonance imaging. Stroke vascular location, i.e. carotidal or vertebrobasilar, was classified on the basis of the neurological examination and/or complementary tests. All of the patients were treated following the recommendations of the Spanish Cerebrovascular Study Group [25]. Accordingly, all of the patients received early antiplatelet therapy except when anticoagulation was recommended (progressing stroke, especially if vertebrobasilar cardioembolic stroke without clinical or radiological signs of a large cerebral infarct, and arterial dissection).

For assessment of SRBDs, an in-house sleep/wake habits and symptoms questionnaire was applied within the first 48-72 h. The questionnaire consisted of 15 items, including snoring, observed apnoea and hypersomnia in different situations, and was related to the time preceding the onset of stroke or TIA [19]. The possible answers were scored as follows: never, rarely, sometimes, often, and always. Daytime sleepiness was also assessed by means of the Epworth Sleepiness Scale [26]. Answers were obtained from the patients, when able to cooperate, and/or through relatives. In all cases, a respiratory sleep study was performed in the ward during the first $48-72 \mathrm{~h}$ after admission, using a portable respiratory recording device (EdenTrace II, Model 3711; EdenTec, Eden Prairie, MN, USA), which had been validated previously using full polysomnography (PSG) [27]. This portable device measures nasal and oral airflow (thermistry), chest wall movements (impedance), cardiac frequency and arterial oxygen saturation $\left(\mathrm{Sa}, \mathrm{O}_{2}\right.$; finger pulse oximetry), snoring and body position. SRBDs were classified as is usual as obstructive or central apnoea, with apnoea considered a cessation of airflow for $\geqslant 10 \mathrm{~s}$ with maintenance of thoracic motion or without any thoracic motion, respectively. A hypopnoea was considered a discernible reduction in airflow or thoracic motion which lasted $>10 \mathrm{~s}$ and was associated with a cyclical dip in $\mathrm{Sa}, \mathrm{O}_{2}$ of $>2 \%$. The CSR pattern was defined as periodic breathing with central apnoea or hypopnoea alternating with hyperpnoea in a crescendo/ decrescendo pattern of $>10 \%$ of the time spent in bed [28]. The apnoea/hypopnoea index (AHI) was calculated, taking into account the time spent in bed with the respiratory recording device (lights out was considered the beginning of the recording and usually initiated between 23:00 and 24:00 $\mathrm{h}$ and terminated between 06:00 and 07:00 h). Manual scoring of these variables was performed in all cases. An experienced scorer, who was blind to the neurological clinical data, performed the scoring. The percentage of night-time with an $\mathrm{Sa}, \mathrm{O}_{2}$ of $<90 \%$ (CT90) was obtained automatically. Full PSG was recorded simultaneously in 10 patients according to the standard criteria of RECHTSCHAFFEN and KALES [29]. Further details of the neurological and sleep study are reported in an earlier paper [19].

The entire cohort was followed by structured telephone interview over a mean period of 22.8 months. A maximal follow-up of 2.5 yrs was established. The patients finished the study because of death, completion of follow-up or elusion of control (change of address, move to nursing home, etc.). The latter were considered censored patients. The outcome event of interest was death and time of survival since the neurological event. Medical records were reviewed for all of the deceased patients wherever possible. The cause of death was classified in accordance with the criteria of SILVER et al. [30], where possible, as cardiovascular conditions, stroke (including acute complications or recurrent stroke), pulmonary disease, others or unknown.

\section{Statistical analysis}

The descriptive analysis comprised arithmetic mean, SD and percentage of cases observed. Survival rates were calculated using the Kaplan-Meier method. Univariate and multivariate survival analyses were performed by means of Cox's proportional-hazards model. All of these variables with a univariate significance of $<0.5$ and no zeros in crosstabulation with dead status were considered for the multivariate model estimation. Covariates were selected in a stepwise procedure using the maximum likelihood ratio (entry criterion $\mathrm{p}<0.05$ (Chi-squared test)). All of the variables entered into the model which did not meet the criteria for remaining in ( $p>0.1$ (Chi-squared test)) were removed in a step-down phase. All p-values were based on two-sided testing.

\section{Results}

Table 1 shows the baseline data from the 161 patients who met the criteria of first ever stroke or TIA. Patients were aged $72 \pm 9$ yrs and had a body mass index (BMI) of $26.6 \pm 3.9 \mathrm{~kg} \cdot \mathrm{m}^{-2}$, an AHI of $21.2 \pm 15.7$ and Epworth Sleepiness Scale score of $4.8 \pm 3.3$. The stroke subtype was classified as ischaemic stroke in $112(69.6 \% ; 44$ lacunar, 34 thrombotic, 25 cardioembolic, seven essential and two unusual) cases, TIA in $39(24.2 \%)$ and intraparenchymatous haemorrhagic stroke in $10(6.2 \%)$. The baseline mean Barthel index score was $75.5 \pm 25.7$ and Canadian scale score 7.9 \pm 2.2 .

Snoring was reported as occurring "never or rarely" in 42 (26.1\%) patients, "sometimes" in 51 (31.7\%) and "often or always" in $61(37.8 \%)$, and no answer was obtained in seven cases. Considering different cut-off points for AHI, $116(72 \%)$ patients had an AHI of $>10,76(47.2 \%)$ an AHI of $>20,45$ $(28 \%)$ an AHI of $>30,19(11.2 \%)$ an AHI of $>40$ and eight $(5 \%)$ an AHI of $>50$. These respiratory events were mainly

Table 1.-Baseline clinical and sleep polysomnographic parameters

\begin{tabular}{lc}
\hline Demographics & \\
Patients n & 161 \\
Age yrs & $72 \pm 9$ \\
BMI kg.m ${ }^{-2}$ & $26.6 \pm 3.9$ \\
ESS score & $4.8 \pm 3.3$ \\
Sleep parameters & \\
AHI & $21.2 \pm 15.7$ \\
CAI & $5.6 \pm 10.1$ \\
CSR n $(\%)$ & $42(26.1)$ \\
AHI $>10 \mathrm{n}(\%)$ & $116(72)$ \\
AHI $>30 \mathrm{n}(\%)$ & $45(28)$ \\
CT 90 & $7.8 \pm 15.7$ \\
\hline
\end{tabular}

Data are presented as mean \pm SD unless otherwise indicated. BMI: body mass index; ESS: Epworth Sleepiness Scale; AHI: apnoea/hypopnoea index; CAI: central apnoea index; CSR: Cheyne-Stokes respiration (periodic breathing with central apnoea or hypopnoea alternating with hyperpnoea in a crescendo/decrescendo pattern for $>10 \%$ of time spent in bed [28]); CT90: percentage of night-time with an arterial oxygen saturation of $<90 \%$. 
obstructive in $84(52.2 \%)$ cases and predominantly central in $62(38.5 \%)$, the remaining $15(9.3 \%)$ showing no predominant pattern. Of the cases with predominantly central events, 37 ( $23 \%$ of the whole group) patients were pure central (all apnoeic events being central). The mean CT90 was 7.8 \pm 15.7 (table 1).

In accordance with brain computed tomography scan and/ or magnetic resonance imaging results, a parenchymatous location was determined in 97 of the 122 patients with established stroke. In the remaining 25 , neuroimaging results were negative. Thus, 81 (50.3\% of the whole group) patients exhibited a hemispheric location, $13(8.1 \%)$ brainstem and three $(1.9 \%)$ cerebellar. The carotid artery territory was involved in $84(52.2 \%)$ and the vertebrobasilar in $39(24.2 \%)$ patients.

The mean duration of follow-up was 22.8 months (range 0.4-32 months). Overall, mortality occurred in 22 of the 161 cases, the mortality rate being $13.7 \%$. The wide range of follow-up times was mainly due to the fact that patients died over a wide range of time. Two patients died within the first 6 months, one of them 2 weeks after stroke event onset, three died during the next 6 months, 12 between 12 and 18 months, and the remaining five between 18 and 24 months. Vascular disease was the major underlying cause of death, accounting for $63.6 \%$ of the deaths. Cerebrovascular disease accounted for $50 \%$ of the deaths (the earliest one was probably caused by transtentorial herniation, and the remaining 10 were due to recurrent acute stroke during follow-up). Cardiovascular conditions were the cause of death in three cases, pulmonary causes in three, septicaemia in two and intestinal occlusion in one. An unknown cause of death was registered in two cases. Antiplatetet or anticoagulant therapy was established, following the recommendations of the Spanish Cerebrovascular Study Group [25], in 128 of the 139 survivors and 21 of the 22 patients who died.

\section{Univariate analysis}

Table 2 shows the results of the univariate analysis considering demographic variables (sex, age and BMI) and known cerebrovascular risk factors. Only significant ones and those with a p-value of $<0.5$ are included in the table. Survivors were significantly younger than patients who died. Diabetes and a history of cardiac insufficiency were related to a poorer outcome $(\mathrm{p}=0.05)$. Ischaemic heart disease and chronic obstructive pulmonary disease also showed a tendency to be more frequent in patients who died.

It was not possible to demonstrate any influence of parenchymatous location on mortality in the univariate approach. Stroke subtype exerted no significant influence on mortality in the present patients.

Antiplatelet or anticoagulant therapy was not regarded by the univariate analysis as significant and was therefore not included in the multivariate regression (table 2).

Sleep respiratory parameters are included in table 3 . The AHI was significantly lower in survivors, being associated

Table 2. - Univariate analysis: clinical parameters ${ }^{\#}$

\begin{tabular}{|c|c|c|c|c|}
\hline & \multicolumn{2}{|c|}{ Vital status } & \multirow[t]{2}{*}{ Hazard ratio $(95 \% \mathrm{CI})$} & \multirow[t]{2}{*}{ p-value } \\
\hline & Alive & Dead & & \\
\hline Males/females $n$ & $72 / 67$ & $10 / 12$ & & \\
\hline Age yrs & $71.0 \pm 9.4$ & $77.3 \pm 7.04$ & $1.08(1.02-1.15)$ & 0.004 \\
\hline $\mathrm{BMI} \mathrm{kg} \cdot \mathrm{m}^{-2}$ & $26.7 \pm 3.8$ & $26.2 \pm 4.5$ & $1.01(0.89-1.14)$ & 0.81 \\
\hline Hypertension $n$ & 83 & 11 & $0.77(0.33-1.78)$ & 0.54 \\
\hline Diabetes $n$ & 35 & 10 & $2.30(0.99-5.33)$ & 0.05 \\
\hline IHD n & 19 & 6 & $2.22(0.86-5.69)$ & 0.09 \\
\hline Arrhythmia $n$ & 29 & 8 & $1.88(0.78-4.48)$ & 0.15 \\
\hline Cardiac insufficiency $\mathrm{n}$ & 5 & 3 & $3.31(0.97-11.23)$ & 0.05 \\
\hline COPD $\mathrm{n}$ & 9 & 4 & $2.49(0.84-7.38)$ & 0.09 \\
\hline Smoking $\mathrm{n}$ & 23 & 3 & $0.79(0.23-2.67)$ & 0.70 \\
\hline Alcohol abuse $n$ & 4 & 1 & $1.15(0.15-8.62)$ & 0.88 \\
\hline Dyslipaemia $n$ & 30 & 1 & $0.19(0.02-1.44)$ & 0.11 \\
\hline Barthel index & $76.5 \pm 25.3$ & $68.4 \pm 27.7$ & $0.99(0.97-1.00)$ & 0.2 \\
\hline Canadian scale & $8.06 \pm 2.16$ & $7.3 \pm 2.7$ & $0.88(0.74-1.00)$ & 0.1 \\
\hline Therapy $\mathrm{n}$ & 128 & 21 & $1.72(0.23-12.8)$ & 0.59 \\
\hline
\end{tabular}

Data are presented as mean \pm SD or as absolute numbers. CI: confidence interval; BMI: body mass index; IHD: ischaemic heart disease; COPD: chronic obstructive pulmonary disease. ${ }^{\#}$ : clinically relevant and/or $\mathrm{p}<0.05 ;{ }^{\uparrow}$ : antiplatelet and anticoagulant.

Table 3. - Univariate analysis: sleep study

\begin{tabular}{lcccc}
\hline & \multicolumn{3}{c}{ Vital status } & Hazard ratio $(95 \%$ CI $)$ \\
\cline { 2 - 3 } & Alive & Dead & \\
\hline Subjects n & 139 & 22 & & \\
AHI & $19.5 \pm 13.9$ & $32.4 \pm 21.3$ & $1.02(1.01-1.04)$ & 0.002 \\
AI & $12.6 \pm 16.3$ & $10.02 \pm 11.7$ & $1.01(0.98-1.04)$ & 0.36 \\
CAI & $5.2 \pm 9.4$ & $8 \pm 13.7$ & $1.01(0.99-1.03)$ & $0.193)$ \\
CT90 & $6.9 \pm 14$ & $13.5 \pm 23.8$ & $2.86(1.23-6.60)$ & 0.19 \\
CSR n (\%) & $31(19.2)$ & $11(50)$ & 0.01 \\
\hline
\end{tabular}

Data are presented as mean $\pm \mathrm{SD}$. AHI: apnoea/hypopnoea index; AI: apnoea index; CAI: central apnoea index; CT90: percentage of night-time with an arterial oxygen saturation of $<90 \%$; CSR: Cheyne-Stokes respiration (periodic breathing with central apnoea or hypopnoea alternating with hyperpnoea in a crescendo/decrescendo pattern for $>10 \%$ of time spent in bed [28]). \#: using portable respiratory recording device. 


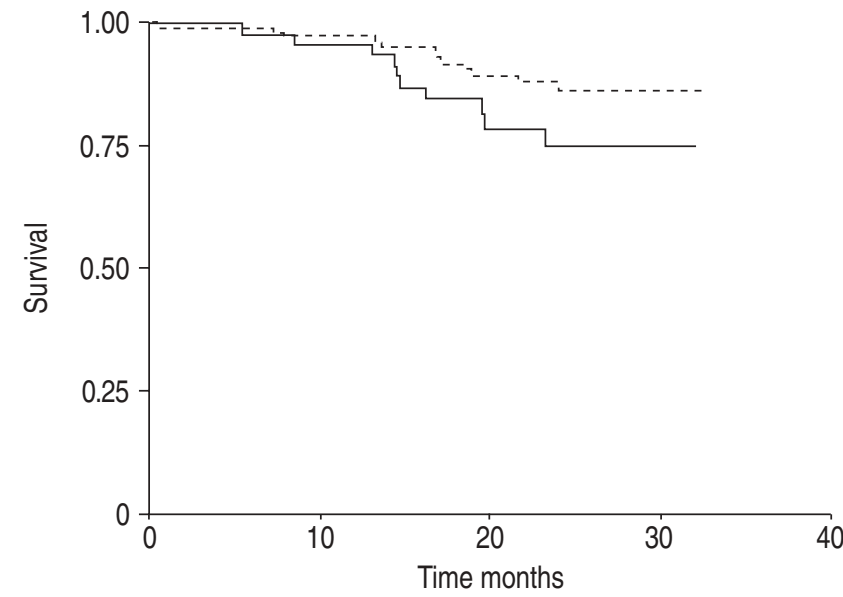

Fig. 1.-Kaplan-Meier survival estimates in patients with an apnoea/ hypopnoea index (AHI) of <30 (----) and in those with an AHI of $\geqslant 30$ ( - ). Greater mortality is evident in patients with an AHI above the cut-off point of 30 .

with mortality. CSR also occurred significantly less in survivors, i.e. $50 \%$ of the patients who died were classified as having this breathing pattern. The mean apnoea index was $10.02 \pm 11.7$ in survivors and $12.6 \pm 16.3$ in patients who died (hazard ratio $1.01 ; \mathrm{p}=0.3(\mathrm{NS})$ ) (table 3 ). In order to ascertain whether the high occurrence of central phenomena could be related to the extent of the neurological lesion, lacunar strokes were regarded as smaller lesions than nonlacunar strokes, but no difference was found in the number of central apnoeas $(4.7 \pm 10.5$ in lacunar and $5.9 \pm 8.9$ in nonlacunar strokes ( $\mathrm{p}=0.47$ (t-test)) or the presence of CSR ( $\mathrm{p}=0.32$ (Chi-squared test)). Although a tendency to a lower score in the Barthel Index and Canadian scale was observed in patients who died, it was not significant (table 2). No correlation was found between the presence of SRBDs and Barthel Index or Canadian scale scores.

From a univariate perspective, it was found that age and AHI were factors with a significant impact on mortality. Figure 1 illustrates differences in survival obtained for an AHI cut-off point of 30, which exerts an influence on mortality.

\section{Multivariate analysis}

Table 4 shows the results of the multivariate analysis, which included four independent variables associated with mortality: 1) age, with a hazard ratio of 1.14 (95\% confidence interval 1.06-1.22), corresponding to an increase in mortality of $14 \% \cdot \mathrm{yr}^{-1}$; 2) AHI, with a hazard ratio of $1.05(1.01-1.08)$, which implies a 5\% increase in mortality risk for each unit of increase in AHI; 3) middle cerebral artery involvement

Table 4.-Cox's proportional-hazards model (multivariate analysis)

\begin{tabular}{lcc}
\hline & Hazard ratio $(95 \% \mathrm{CI})$ & p-value \\
\hline Age & $1.14(1.06-1.21)$ & 0.000 \\
AHI & $1.05(1.01-1.08)$ & 0.004 \\
MCA involvement & $2.86(1.04-7.84)$ & 0.04 \\
Coronary disease & $3.25(1.05-10.03)$ & 0.04 \\
\hline
\end{tabular}

CI: confidence interval; AHI: apnoea/hypopnoea index; MCA: middle cerebral artery. (hazard ratio $2.86(1.04-7.84)$ ); and 4) the presence of ischaemic heart disease (hazard ratio 3.25 (1.05-10.03)).

\section{Discussion}

Earlier studies indicate that SRBDs are frequent in patients with stroke $[8-11,19,31,32]$. Furthermore, in the present study, the AHI appears to be an independent prognostic factor in such patients since mortality increases in proportion to the AHI.

Other authors have demonstrated an increase in sleep apnoea patients' mortality depending on AHI. HE et al. [15] found that those with an apnoea index of $>20$ exhibited higher mortality than those with a lower apnoea index. This mortality was due to vascular diseases, either myocardial infarction or stroke. PARTINEN et al. [16] also detected a higher crude vascular mortality in patients with SRBDs. LAVIE et al. [17], in a prospective study of middle-aged sleep apnoea patients, and LINDBERG et al. [18], in a populationbased study, also demonstrated that apnoea index or snoring and excessive daytime sleepiness were predictors of excessive mortality, but both failed to show increased cardiovascular mortality among older patients, aged $>60$ yrs. In contrast to these data, the present study shows a relationship between AHI and mortality in patients with a mean age of 72 yrs. This apparent discrepancy is probably due to the fact that the present patients have already suffered a stroke or TIA, and, therefore, a specific group of patients, in which age is a known prognostic factor, are being faced. Moreover, this fact could reinforce the idea that, when stroke has occurred, SRBDs can be an independent prognostic factor adjusted for age, another important prognostic factor.

There are few data showing the influence of SRBDs on mortality in stroke patients. Despite the reduced amount of data from the 22 dead patients, to the present authors' knowledge, this is the largest group of first ever stroke or TIA patients $(n=161)$ investigated using a sleep study and followup for a mean period of 2 yrs. There are few communitybased studies of long-term prognosis after acute stroke. Taking into account some data published regarding long-term survival after first ever stroke, DENNIS et al. [33] showed an absolute risk of death of $15 \%$ over the first year after stroke. Other articles included patients with recurrent stroke, yielding a poorer prognosis [34]. Death in 22 of 161 patients represents a mortality of $13.6 \%$ over a mean period of $2 \mathrm{yrs}$, which is lower than expected [35]. This could be attributed to the inclusion of TIA patients $(\mathrm{n}=39)$, with a better prognosis, and a low proportion of haemorrhagic stroke in the present setting, which has a worse prognosis [36], as well as to the entry criteria, since patients with a previous stroke were excluded [19]. Thus, it is difficult to put the present group of patients in perspective in terms of general stroke mortality. DYKEN et al. [9], in a study including 24 patients with stroke, described a high incidence of SAS, with a 4-yr mortality rate of $20.8 \%$. They reported that all patients who died had obstructive sleep apnoea. An earlier report also found that snorers with stroke were more likely to die within a period of 6 months than nonsnorers [37]. Another report demonstrated a poorer functional outcome in patients with stroke and obstructive sleep apnoea [38]. The present study is the first to show that AHI is an independent prognostic factor associated with mortality in patients with stroke.

Another possible limiting factor in the present study is the variability in the duration of follow-up of the patients. However, if the survival distribution is analysed, it is apparent that the wide range of follow-up was mainly due to the fact that patients died over a wide period of time. All of the 
patients who died did so within the first 24 months; the first censored patient was not detected until 10 months.

A number of well-identified predictive factors of mortality in stroke were analysed in the present study, considering age or BMI, among others, which could act as confounding factors. The influence of age on the probability of a fatal outcome has also been confirmed by earlier studies [39-43]. The present data demonstrated that age was significantly greater in patients who died than in those who survived on univariate analysis. The multivariate analysis also identified age as an independent factor related to mortality, showing a $14 \% \cdot \mathrm{yr}^{-1}$ increase in mortality. The older the patient the more advanced the atherosclerosis and the higher the frequency of previous or concomitant pathological conditions. Other known prognostic factors, such as diabetes, ischaemic heart disease and cardiac insufficiency, showed a significant or almost significant univariate influence, which is in agreement with other studies [39-42]. However, some recognised prognostic factors failed to show an independent relation with survival, possibly because of the sample size and the low mortality rate.

The stroke subtype showed no significant difference, on univariate or multivariate analysis, between the two groups. Despite the fact that haemorrhage is considered a worsening prognostic factor, there were insufficient patients with haemorrhage to confirm this fact.

An encephalic or brainstem location was not related to survival. Middle cerebral vascular topography was associated with an increase in mortality, since $>50 \%$ of patients who died were thus affected. The presence of a nonlacunar stroke in the territory of the middle cerebral artery was also an independent factor associated with hospital mortality in an earlier study [43]. This is reasonable given that extensive stroke, which has a poorer prognosis, occurs when this vascular territory is involved. Furthermore, middle cerebral artery lesions are also one of the independent factors associated with mortality, with a hazard ratio of 2.85 , which account for an almost three-fold increase in the probability of death and early death.

Ischaemic heart disease is another independent factor that is related to mortality in the present study, in agreement with previous data [39-44].

However, the most interesting data in the present study are the portable respiratory recording device results. This is simple and validated device [27], which can easily be used at the bedside. This is of great help to such patients, whose management is of considerable complexity, especially in the acute phase, when cognitive impairment and/or physical disability is present. The availability of such data during the acute phase may have prognostic implications not only for survival but also for improving outcome, since the application of nasal continuous positive airway pressure could reduce the risk of hypertension and stroke in patients with SRBDs and perhaps improve early rehabilitation potential after stroke $[45,46]$. In the present authors' experience, performing full PSG in the sleep laboratory in such patients is not easy, with the result that the opportunity to perform a respiratory sleep study would be lost in some cases. In the study of BASSETTI and ALDRICH [11], full PSG was not feasible in $37.5 \%$ of patients, when performed at a mean time of 9 days. Although the present respiratory recording device has not been validated for the analysis of central apnoeas or CSR, their definition was based on respiratory parameters, specifically a combination of thoracoabdominal movements and a thermistor [28].

Thus, AHI was significantly higher in patients who died. A tendency for more central apnoeas was observed in the group who died, as well as a significantly more frequent CSR pattern, present in $50 \%$. The latter could be related to greater extension of the stroke, which could imply a poorer prognosis, although a satisfactory explanation is not available for this observation since this hypothesis has not been proved. Nevertheless, central events accounted for only a small number of the total respiratory events. Only AHI, obstructive events being the predominant factor, was independently associated with mortality. Although the magnitude of this relationship may seem low (hazard ratio 1.05), it is significant, and establishing such a relation is enough to suggest that the higher the AHI the higher the probability of death, adjusting for the rest of the variables. Reduction in cerebral blood flow in the middle cerebral artery during obstructive events could explain how SRBDs could adversely affect prognosis in such patients. In this sense, most patients died because of CVD or cardiovascular disease. Therefore, SRBDs could be regarded not only as a probable risk factor but also as a prognostic factor.

In conclusion, the present study on mortality risk in patients with stroke suggests that sleep-related breathing disorders are an independent prognostic factor related to mortality. These data require placing into clinical perspective, especially considering the fact that two recent studies suggest a possible positive effect of nasal continuous positive airway pressure in stroke patients $[45,46]$.

\section{References}

1. Koskenvuo M, Kapiro J, Telakivi T, Partinen M, Heikkila $\mathrm{K}$, Sarna S. Snoring as a risk factor for ischaemic heart disease and stroke in men. BMJ 1987; 294: 16-19.

2. Sirne S, Palazzi S, Zucconi M, Chierchia S, Strambi LF Habitual snoring as a risk factor for acute vascular disease. Eur Respir J 1993; 6: 1357-1361.

3. Palomäki H, Partinen M, Juvela S, Kaste M. Snoring and the risk of ischaemic brain infarction. Stroke 1991; 22: 1021-1025.

4. Palomäki H, Partinen M, Erkinjuntti T, Kaste M. Snoring, sleep apnea syndrome and stroke. Neurology 1992; 42: Suppl. 6, 75-82.

5. Lee MC, Klassen AC, Hearney LM, Perch JA. Respiratory rate and pattern disturbances in acute brain stem infarction. Stroke 1976; 7: 382-385.

6. Levin BE, Margolis G. Respiration secondary to a unilateral brain stem infarction. Ann Neurol 1977; 1: 583-586.

7. Askenasy JM, Goldhamer I. Sleep apnea as a feature of bulbar stroke. Stroke 1988; 19: 637-639.

8. Bassetti C, Aldrich MS, Chervin RD, Quint D. Sleep apnea in patients with transient ischemic attack and stroke: a prospective study of 59 patients. Neurology 1996; 47: 1167-1173.

9. Dyken ME, Somers VK, Yamada T, Ren ZY, Zimmerman MB. Investigating the relationship between stroke and obstructive sleep apnea. Stroke 1996; 27: 401-407.

10. Mohsenin V, Valor R. Sleep apnea in patients with hemispheric stroke. Arch Phys Med Rehabil 1995; 76: 71-76.

11. Bassetti C, Aldrich MS. Sleep apnea in acute cerebrovascular disease: final report in 128 patients. Sleep 1999; 22: 217-223.

12. Wright J, Johns R, Watt I, Melville A, Sheldon T. Health effects of obstructive sleep apnoea and the effectiveness of continuous airway pressure: a systematic review of the research evidence. $B M J 1997 ; 314: 851-860$.

13. Hung J, Whitford EG, Pearsons RW, Hillman DR. Association of sleep apnea with myocardial infarction in men. Lancet 1990; 336: 251-264.

14. Stradling JR. Sleep apnea and systemic hypertension. Thorax 1989; 44: 984-989.

15. He J, Kryger MH, Zorick FJ, Conway W, Roth T. Mortality and apnea index in obstructive sleep apnea: experience in 385 male patients. Chest 1988; 94: 9-14.

16. Partinen M, Jamieson A, Guilleminault C. Long-term 
outcome for obstructive sleep apnea syndrome patients. Mortality. Chest 1988; 94: 1200-1204.

17. Lavie P, Herer P, Peled R, et al. Mortality in sleep apnea patients: a multivariate analysis of risk factors. Sleep 1995; 18: $149-157$.

18. Lindberg E, Janson C, Svärdsudd K, Gislason T, Hetta J, Boman G. Increased mortality among sleepy snorers: a prospective population based study. Thorax 1998; 53: 631-637.

19. Parra O, Arboix A, Bechich S, et al. Time course of sleeprelated breathing disorders in first-ever stroke or transient ischemic attack. Am J Respir Crit Care Med 2000; 161: 375-380.

20. Netzer N, Werner P, Jochums I, Lehmann M, Strohl K. Blood flow of the middle cerebral artery with sleepdisordered breathing. Stroke 1998; 29: 87-93.

21. Arboix A, Massons J, Oliveres M, García L, Titus F. Análisis de 1000 pacientes consecutivos con enfermedad cerebrovascular aguda. Registro de patología cerebrovascular de La Alianza-Hospital Central de Barcelona. Med Clin (Barc) 1993; 191: 281-285.

22. Arboix A, Morcillo C, García-Eroles L, Oliveres M, Massons J, Targa C. Different vascular risk factor profiles in ischemic stroke subtypes: a study from the "Sagarat Cor Hospital of Barcelona Stroke Registry". Acta Neurol Scand 2000; 102: 264-270.

23. Cote R, Hachinski VC, Shurvell BL, Norris JW, Wolfson C. Canadian Neurological Scale. A preliminary study in acute stroke. Stroke 1986; 17: 731-737.

24. Special report from the National Institute of Neurological Disorders and Stroke. Classification of cerebrovascular disease. Stroke 1990; 21: 637-676.

25. Grupo de Estudio de las Enfermedades Cerebrovasculares de la Sociedad Española de Neurología. Manejo del Paciente con Enfermedad Vascular Cerebral Aguda. Recomendaciones 1992. Barcelona, Edos Ed, 1992.

26. Johns MW. A new method for measuring daytime sleepiness: the Epworth Sleepiness Scale. Sleep 1991; 14: 540-545.

27. Parra O, García-Esclassans N, Montserrat JM, et al. Should patients with sleep apnoea/hypopnoea syndrome be diagnosed and managed on the basis of home sleep studies? Eur Respir J 1997; 10: 1720-1724.

28. Nachtmann A, Siebler M, Rose G, Sitzer M, Teimetz H. Cheyne-Stokes respiration in ischemic stroke. Neurology 1995; 45: 820-821.

29. Rechtschaffen A, Kales A. A manual of standardized terminology, techniques and scoring system for sep stages of human subjects. NIH publication no. 204. Bethesda, MD, 1968.

30. Silver FL, Norris JW, Lewis AJ, Hachinsky VC. Early mortality following stroke: a prospective review. Stroke 1984; 15: 492-496.

31. Bassetti C, Aldrich MS, Quint D. Sleep-disordered breathing in patients with acute supra- and infratentorial strokes. A prospective study of 39 patients. Stroke 1997; 28: 1765-1772.

32. Kapen S, Park A, Goldberg J, Whynter J. The incidence and severity of obstructive sleep apnea in ischaemic cerebrovascular disease. Neurology 1991; 41: Suppl. 1, 125.

33. Dennis MS, Burn PS, Sandercock PAG, Bamford JM, Wade DT, Warlow CP. Long-term survival after first-ever stroke: the Oxfordshire Community Stroke Project. Stroke 1993; 24: 796-800.

34. van Straten A, Reitsma JB, Limburg M, van den Bos GAM, de Haan RJ. Impact of stroke type on survival and functional health. Cerebrovasc Dis 2001; 12: 27-33.

35. Arboix A, García-Eroles L, Comes E, et al. Predicting spontaneous early neurological recovery after acute ischemic stroke. Eur J Neurol 2003; 10: 429-435.

36. Arboix A, Massons J, García-Eroles L, Oliveres M, Targa C. Diabetes is an independent risk factor for in-hospital mortality from acute intracerebral hemorrhage. Diabetes Care 2000; 23: 1527-1532.

37. Spriggs DA, French JM, Murdy JM, Curles RH, Bates D, James OFW. Snoring increases the risk of stroke and adversely affects prognosis. $Q J$ Med 1992; 84: 555-562.

38. Good DC, Henkle JQ, Geller D, Welsh J, Verhulst S. Sleep disordered breathing and poor functional outcome after stroke. Stroke 1996; 27: 252-259.

39. Sacco R, Shi T, Zamanillo MC, Kargman DE. Predictors of mortality and recurrence after hospitalized cerebral infarction in an urban community: the Northern Manhattan Stroke Study. Neurology 1994; 44: 626-634.

40. Westling B, Norrving B, Thornguen M. Survival following stroke. A prospective population based study of 438 hospitalized cases with prediction according to subtype, severity and age. Acta Neurol Scand 1990; 81: 457-463.

41. Fiorelli M, Alperovitch A, Argentino C, et al. Prediction of long-term outcome in the early hours following acute ischemic stroke. Arch Neurol 1995; 52: 250-255.

42. Chambers BR, Norris JW, Shunell BL, Hachinski V. Prognosis of acute stroke. Neurology 1987; 37: 221-225.

43. Arboix A, García-Eroles L, Massons J, Oliveres M. Clinical predictors of inhospital mortality in 986 consecutive patients with first-ever stroke. Cerebrovasc Dis 1996; 6: 161-165.

44. Hart CL, Hole DJ, Smith GD. Comparison of risk factors for stroke incidence and stroke mortality in 20 years of follow-up in men and women in the Renfrew/Paisley Study in Scotland. Stroke 2000; 31: 1893-1896.

45. Wessendorf TE, Wang YM, Thilmann AF, Sorgenfrei U, Konietzko N, Teschler H. Treatment of obstructive sleep apnea with nasal continuous positive airway pressure in stroke. Eur Respir J 2001; 18: 623-629.

46. Sandberg O, Franklin KA, Bucht G, Eriksson S, Gustafson Y. Nasal continuous positive airway pressure in stroke patients with sleep apnea: a randomized treatment study. Eur Respir J 2001; 18: 630-634. 